\title{
Influência do lenho de tração nas propriedades físicas da madeira de Eucalyptus sp.
}

\author{
Thiago Campos Monteiro ${ }^{{ }^{*}}$, Renato Vieira da Silva ${ }^{2}$, José Tarcísio Lima ${ }^{1}$, Edy Eime \\ Pereira Baraúna², Duam Matosinhos de Carvalho², Marcelle Teodoro Lima ${ }^{2}$
}

${ }^{1}$ Departamento de Ciências Florestais/Laboratório de Ciência e Tecnologia da Madeira; Universidade Federal de Lavras; 37200-000; Lavras- MG- Brasil. ${ }^{2}$ Curso de Engenharia Florestal; Universidade Federal do Tocantins; 77402-970; Gurupi - TO-Brasil.

\begin{abstract}
The reaction wood is formed in an attempt to remain upright tree in response to the action of forces such as winds, irregular crown or slope of the land that tend to incline it. In hardwoods, as in Eucalyptus, this type of wood is called tension wood and occurs in the region of the stem facing the face of force application. Indicative of the presence of this type of wood is the high shrinkage and basic density compared to normal wood. Once the basic density and shrinkage are parameters for determining the quality of the wood, this study aimed to evaluate the variation of basic density and shrinkage of opposite and tension wood along the radius in four species of Eucalyptus sp. Four tree species Eucalyptus camaldulensis, Eucalyptus maculata, Eucalyptus pilularis and Eucalyptus urophylla, with 32 years of age, were taken from an experimental planting of the Federal University of Lavras. Specimens were made to represent the diametrical variation of the opposite of tension wood in disks cut at the dbh. The results indicate that the properties of radial, tangential and volumetric shrinkage, coefficient of anisotropy and basic density did not differ statistically between the tension and opposite wood.
\end{abstract}

Key words: Basic density, shrinkage, reaction wood, pith-bark direction

\section{INTRODUÇÃO}

As árvores são estruturas complexas como todos os seres biológicos e apresentam variações das propriedades anatômicas, físicas, mecânicas e químicas tanto no sentido longitudinal quanto no sentido radial. Para as propriedades físicas a variação no sentido radial é maior do que no sentido longitudinal (Cruz et al., 2003). A intensidade da variação no lenho da árvore ocorre, segundo Zobel e Jett (1995), devido aos fatores genéticos, ambientais e a interação entre estes dois fatores.

Entre os fatores ambientais responsáveis pelas modificações no lenho podem-se citar o clima, o solo, a topografia entre outros. Em árvores plantadas em encostas e susceptíveis a ação de ventos, estas tendem a formar um lenho diferenciado, denominado lenho de reação. Além destes fatores externos, a ocorrência de copa assimétrica na árvore, e a massa dos galhos tendem a provocar a formação deste lenho de reação. Trabalhos como Simpson e Tenwolde
(1999), apresentam que a formação deste tipo de lenho é freqüentemente associada à inclinação ou tortuosidade do fuste. Este trabalho também cita que o lenho de reação ocorre como uma resposta natural da árvore para retornar os seus membros ou fuste para a posição normal durante ou após a ação de forças.

Nas coníferas o lenho de reação é conhecido como lenho de compressão e nas folhosas, como os Eucalyptus, é chamado de lenho de tração. Geralmente o lenho de compressão ocorre do lado inferior do fuste inclinado e nas folhosas do lado superior do lenho inclinado (Simpson e Tenwolde, 1999). A ocorrência do lenho de reação é mais susceptível em algumas espécies do que em outras e as propriedades físicas, químicas e mecânicas deste lenho diferem do lenho normal (Green et al., 1999).

Uma forma de observar a presença do lenho de reação é o crescimento excêntrico da medula na tora, ou seja, o deslocamento da medula do seu centro geométrico sugere a presença do lenho de

E-mail para correspondência: tcmforest@yahoo.com 
tração ou compressão (Simpson e Tenwolde, 1999). Afirmativa também encontrada por Ferreira et al. (2008), que estudaram os clones de Eucalyptus com dois anos de idade, plantados em locais de baixada, encosta leve e encosta forte e concluiu que o deslocamento da medula indica a formação do lenho de tração. Além da excentricidade da medula, ocorrem também diferenças nas propriedades químicas, anatômicas, físicas e mecânicas neste tipo de lenho quando comparado com um lenho normal. Dentre as características que apresentam destaque pode-se citar o baixo teor de lignina na parede celular das fibras, sendo essa deficiência uma das características do desenvolvimento do lenho de tração (Panshin e De Zeeuw, 1980), a ocorrência de uma camada gelatinosa na parte interna à parede celular secundária, a excessiva contração quando a umidade fica abaixo do ponto de saturação das fibras e o aumento da densidade básica (Simpson e Tenwolde, 1999).

A ocorrência do lenho de reação influencia na utilização da madeira na indústria florestal. Sua presença produz uma superfície irregular quando usinada, suas tábuas apresentam cores contrastantes, com aparência prateada ou escura variando com a espécie, a superfície das tábuas serradas são "lanosas", especialmente quando serradas na condição verde (Simpson e Tenwolde, 1999), defeitos surgem durante a secagem (Ponce e Watai, 1985) e devido a variação do teor de lignina influencia nos setores de celulose e siderúrgico.

O conhecimento da variação da densidade básica do lenho de reação é importante, pois é uma propriedade física de fácil determinação e que apresenta relevante correlação com outras propriedades da madeira (Panshin e De Zeeuw, 1980). Outra característica física citada na literatura para o estudo do lenho de reação é a retratibilidade, que é uma característica relacionada à expansão ou contração da madeira em função da entrada ou saída de água no lenho, sendo influenciada pelos planos da madeira avaliada, se radial, tangencial ou longitudinal (Durlo e Marchiori, 1992).

Os estudos realizados com o lenho de reação comparam este com um lenho normal da mesma espécie, geralmente sem a medula excêntrica. Trabalhos recentes comparando o lenho de reação com o lenho oposto apresentam resultados que não seguem um padrão definido (Ruelle et al., 2007).
Estudos avaliando o lenho de tração em Eucalyptus foram realizados no Brasil, sempre com árvores de pouca idade, abaixo de cinco anos (Sousa, 2004; Ferreira, 2007).

Sendo o gênero Eucalyptus uma espécie muito utilizada no setor produtivo florestal brasileiro, plantado em diversos tipos de terreno, sob a ação de ventos e outros fatores externos, o presente trabalho teve como objetivo avaliar a variação da densidade básica e da retratibilidade dos lenhos de tração e lenho oposto ao longo do raio em quatro espécies de Eucalyptus spp.

\section{MATERIAL E MÉTODOS}

Foram utilizadas quatro árvores do gênero Eucalyptus, sendo das seguintes espécies: Eucalyptus pilularis, Eucalyptus urophylla, Eucalyptus camaldulensis e Eucalyptus maculata. As árvores foram selecionadas entre as eretas, sadias e sem bifurcação retiradas de um plantio experimental, com 32 anos, no campus da Universidade Federal de Lavras (UFLA), em uma área que apresenta leve inclinação.

Os discos foram retirados a 1,3 metros do solo (DAP) de cada uma das árvores e foram levados para o Laboratório de Usinagem da Madeira na UFLA. Foi retirada a porção central de cada disco, contendo a medula e seguindo o sentido da declividade. Em seguida foram determinados os lenhos de tração (lado superior do terreno) e lenho oposto (lado inferior do terreno). Após as marcações, os corpos-de-prova foram confeccionados com as dimensões de 20 x 20 x 30 $\mathrm{mm}$ nos sentidos radial, tangencial e longitudinal, respectivamente, sendo retirados a partir da medula até a proximidade da casca, conforme Figura 1.

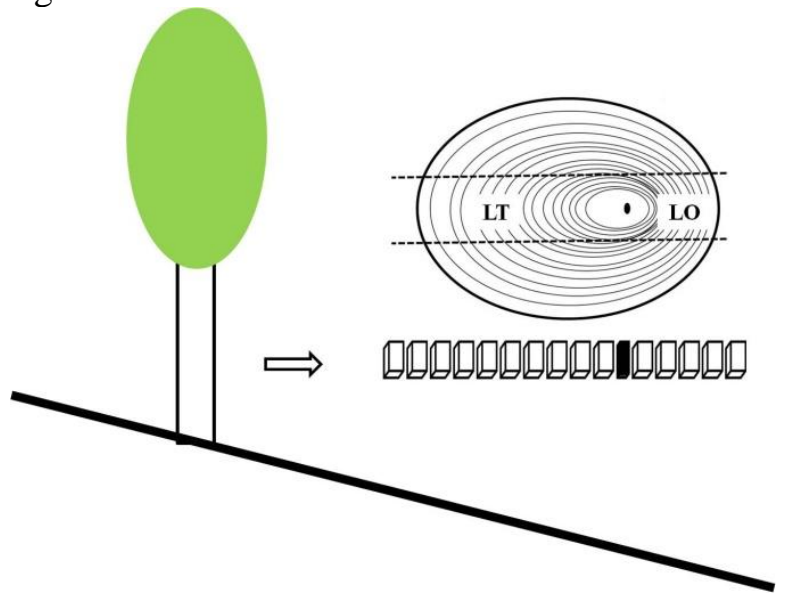


Figura 1- Esquema da retirada de corpos-de-prova para determinação da densidade básica e retratibilidade nos lenhos de tração (LT) e lenho oposto (LO) ao longo do raio nos discos de Eucalyptus.

A excentricidade da medula foi calculada obtendo os diâmetros de cada disco utilizando duas marcações ao longo da extensão diametral. O cruzamento entre as duas marcações foi considerado como o centro geométrico do disco e então obtido a distância da medula até este centro utilizando uma régua com precisão de $1 \mathrm{~mm}$. Em seguida foi calculado a excentricidade da medula utilizado à seguinte equação:

Em que:

$$
\mathrm{EXM}=\frac{\mathrm{Lc}}{\mathrm{dm}} \times 100
$$

EXM = excentricidade da medula,

Lc = distância entre o centro geométrico e a posição real da medula $(\mathrm{cm})$ e

$\mathrm{dm}=$ diâmetro médio do disco $(\mathrm{cm})$.

Para determinar a densidade básica, as amostras foram colocadas em um dessecador com água, e aplicado 30 minutos de vácuo diariamente durante duas semanas. Em seguida a densidade básica dos corpos-de-prova de cada posição foi determinada de acordo com a norma ABNT NBR 11941/2003.

Foi medida a contração radial, tangencial, e volumétrica, desconsiderando a contração no sentido longitudinal. Nas determinações lineares e volumétricas foram utilizados os mesmos corposde-prova usados para densidade básica. A contração radial e tangencial foi medida com paquímetro digital com precisão de $0,01 \mathrm{~mm}$, em locais previamente estabelecidos e demarcados. As medidas foram realizadas com os corpos-de-prova saturados e secos em estufa $\left(103 \pm 2{ }^{\circ} \mathrm{C}\right)$.

A determinação dos volumes para cálculo da contração volumétrica foi pelo método de imersão em mercúrio com as amostras saturadas e após secas em estufa.

Após a coleta dos dados, foi realizada a análise de variância utilizando o software Sisvar.

\section{RESULTADOS E DISCUSSÃO}

Os dados da Tabela 1 apresentam os valores para a excentricidade da medula e os valores médios da retratibilidade e densidade básica das árvores de Eucalyptus sp.

Tabela 1. Excentricidade da medula (EXM) e valores médios em porcentagem para contração tangencial $(\mathrm{Tg})$, radial $(\mathrm{Rd})$, volumétrica $(\mathrm{Vol})$ e resultado médio do coeficiente de anisotropia (CA) e densidade básica $\left(\mathrm{DB} ; \mathrm{g} / \mathrm{cm}^{3}\right)$ para as quatro espécies de Eucalyptus.

\begin{tabular}{lcccccc}
\hline \multicolumn{1}{c}{ Espécie } & EXM \% & \multicolumn{3}{c}{ Retratibilidade \% } & DB g/cm \\
\hline & Média & Tg & Rd & Vol & CA & Média \\
\hline Eucalyptus pilularis & 16,67 & $10,18 \mathrm{a}$ & $7,05 \mathrm{a}$ & $18,09 \mathrm{a}$ & $1,48 \mathrm{~b}$ & $0,696 \mathrm{a}$ \\
Eucalyptus urophylla & 8,39 & $10,43 \mathrm{a}$ & $4,58 \mathrm{~b}$ & $16,20 \mathrm{~b}$ & $2,28 \mathrm{a}$ & $0,571 \mathrm{~b}$ \\
Eucalyptus camaldulensis & 4,26 & $9,10 \mathrm{~b}$ & $6,29 \mathrm{a}$ & $16,05 \mathrm{~b}$ & $1,57 \mathrm{~b}$ & $0,663 \mathrm{ab}$ \\
Eucalyptus maculata & 6,98 & $10,35 \mathrm{a}$ & $7,14 \mathrm{a}$ & $18,13 \mathrm{a}$ & $1,49 \mathrm{~b}$ & $0,750 \mathrm{a}$ \\
\hline
\end{tabular}

Os valores obtidos para excentricidade da medula indicam a presença de medula em todos os discos. No Eucalyptus pilularis nota-se um deslocamento mais acentuado da medula em relação às outras espécies, e no Eucalyptus camaldulensis o deslocamento menos acentuado. Estes valores estão superiores aos obtidos por Ferreira et al. (2008), quando avaliaram clones de Eucalyptus em terrenos de encosta leve e forte. A ocorrência da excentricidade da medula sugere a presença de lenho de tração (Simpson e Tenwolde, 1999; Ferreira et al.,2008).
A retratibilidade apresentou resultados próximos entre as espécies. Das quatro espécies analisadas, apenas uma diferiu estatisticamente para contração tangencial e radial e coeficiente de anisotropia. Duas espécies diferiram estatisticamente para contração volumétrica. Os resultados obtidos nestas espécies são superiores aos encontrados por Cruz et al. (2003), que avaliou clones de Eucalyptus urophylla e Eucalyptus camaldulensis com 5,5 e 10,5 anos, e encontraram valores médios para as contrações radial de $4,9 \%$, para contração tangencial e de $8,9 \%$ e para contração volumétrica 
de 13, 5\%. Em trabalho utilizando Eucalyptus urophylla com 11 anos, originários da China, a contração radial apresentou valores entre 6,08 e $6,81 \%$, a contração tangencial entre 9,26 e 10,25\% (Yi-Qiang et al., 2006). Os valores elevados obtidos nas espécies de Eucalyptus deste trabalho, para contração radial, tangencial e volumétrica são indicativos da presença do lenho de reação. Pois a contração excessiva do lenho de tração está associada ao colapso na maioria das espécies (Panshin e De Zeeuw, 1980). E ainda Wardrop e Dadswell citado por Sousa (2004), encontraram várias explicações a respeito da alta contração no lenho de tração. Estes autores sugerem que a contração pode ser resultado de fissuras desenvolvidas nos espaços intercelulares e que podem ser atribuídas à presença da camada gelatinosa que é frouxa, e devido à natureza higroscópica da camada gelatinosa, que sofre mudanças dimensionais ao secar.

Os coeficientes de anisotropia ficaram classificados como excelente (de 1,2 até 1,5; para utilização nobre em móveis e outros artefatos) para as espécies E. pilularis e E. maculata; como normal $(1,5$ até 2,0$)$ para $E$. camaldulensis e ruim (2,0 ou mais) para E. urophylla (Durlo e Marchiori, 1992). Estes resultados estão próximos dos obtidos por

Cruz et al. (2003), que encontraram para o coeficiente de anisotropia resultado médio igual a 1,91 .

A elevada densidade básica (Tabela 2) pode ser em função da idade das árvores e também um dos indicativos do lenho de reação na madeira estudada. Em madeiras de Eucalyptus sem o relato de lenho de tração a densidade básica para Eucalyptus urophylla varia entre 0,437 e 0,577 $\mathrm{g} / \mathrm{cm}^{3}$ e para Eucalyptus camaldulensis varia entre 0,467 e 0,501 g/cm ${ }^{3}$ (Cruz et al., 2003); em Eucalyptus urophylla com 11 anos originários da
China estes valores foram de $0,47 \mathrm{~g} / \mathrm{cm}^{3}$ (Yi-Qiang et al., 2006).

A Figura 2 apresenta a variação da densidade básica $\left(\mathrm{g} / \mathrm{cm}^{3}\right)$ ao longo do lenho de tração e do lenho oposto para as quatro árvores.

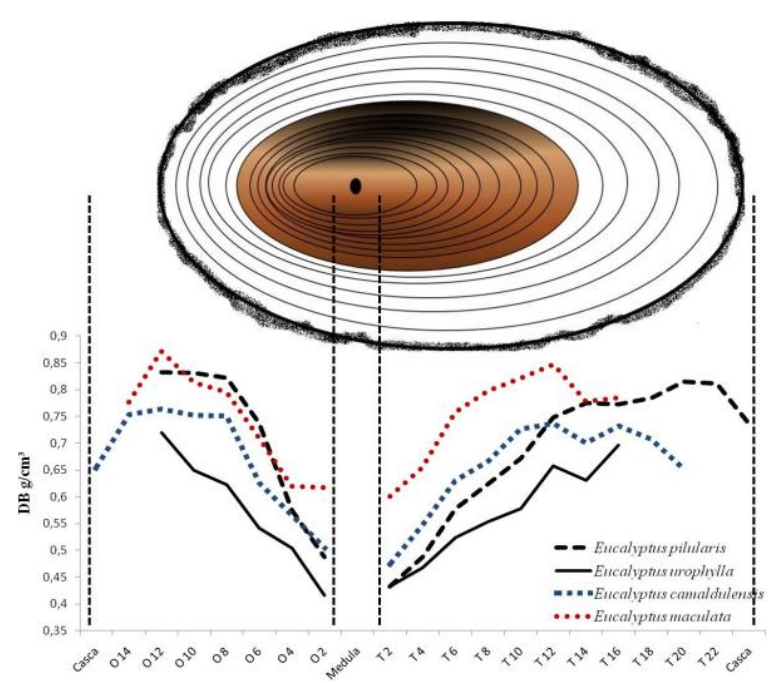

Figura 2- Comportamento da densidade básica (DB $\mathrm{em} \mathrm{g} / \mathrm{cm}^{3}$ ) no setido transversal nos lenhos oposto (O2 até O14) e de tração (T2 até T22) para as quatro árvores de Eucalyptus.

Nota-se que a densidade básica aumenta no sentido medula casca tanto no lenho de tração quanto no lenho oposto, tendo em algumas espécies um decréscimo próximo à casca. Este comportamento do aumento da densidade básica no sentido medula casca é descrito na literatura por diversos autores (Panshin e De Zeeuw, 1980; Tomazello Filho, 1985; Cruz et al., 2003; Ferreira, 2007).

A Tabela 2 apresenta o resumo da analise de variância comparando os lenhos de tração e oposto para as contrações radial, tangencial e volumétrica, para o coeficiente de anisotropia e para a densidade básica (Tabela 2).

Tabela 2. Resumo da análise de variância da contração radial (Rd), contração tangencial (Tg), contração volumétrica (Vol), coeficiente de anisotropia (CA) e densidade básica (DB) dos lenhos de tração e oposto das quatro espécies de Eucalyptus

\begin{tabular}{lcccccc}
\hline \multirow{2}{*}{ Fonte de variação } & \multirow{2}{*}{ GL } & Rd & Tg & Vol & CA & DB \\
\cline { 3 - 7 } & & QM & QM & QM & QM & QM \\
\hline Lenho & 1 & $1,0616^{\text {ns }}$ & $0,1571^{\text {ns }}$ & $0,7397^{\text {ns }}$ & $0,0188^{\text {ns }}$ & $0,0015^{\text {ns }}$ \\
Erro & 63 & 4,5415 & 1,4571 & 3,1290 & 0,1908 & 0,0144 \\
Total corrigido & 64 & & & & & \\
\hline
\end{tabular}

Em que: $n s-$ não significativo a $5 \%$. 
Verifica-se que não ocorreu diferença significativa, em nível de $95 \%$ de probabilidade, entre os lenhos de tração e oposto para todas as propriedades avaliadas. Este comportamento também foi obtido por Ferreira (2007), que avaliou os lenhos de tração e oposto em clones de Eucalyptus com dois anos de idade, plantados em três diferentes topografias, não constatando também diferença significativa entre os lenhos para a densidade básica. Já para a contração volumétrica obteve diferença significativa para os clones plantados apenas na encosta forte. Já Sousa (2004), obteve diferença significativa entre os lenhos de tração e o lenho oposto, em um híbrido de Eucalyptus grandis, com quatro anos de idade, avaliando a densidade básica e a contração volumétrica.

No entanto o comportamento das propriedades físicas entre os lenhos de tração e o lenho oposto não apresentam comportamento definido. Conforme Ruelle et al. (2007), que avaliou a densidade básica do lenho de tração e oposto em dez espécies nativas da floresta tropical e em apenas cinco espécies foi obtido diferença significativa entre os lenhos. Das cinco espécies, três (Ocotea guyanensis, Carapa procera, Qualea rosea) apresentaram a densidade do lenho de tração maior que o lenho oposto e duas (Miconia fragilis, Virola surinamensis) apresentaram a densidade do lenho oposto maior que o lenho de tração.

Observando a tendência entre os lenhos de tração e oposto das quatro espécies de Eucalyptus, observase que não ocorreu comportamento definido entre os lenhos para as propriedades físicas avaliadas (Tabela 3).

Tabela 3. Valores médios dos lenhos de tração e lenho oposto das quatro espécies de Eucalyptus para a contração radial (Rd), contração tangencial $(\mathrm{Tg})$, contração volumétrica (Vol), coeficiente de anisotropia (CA) e densidade básica (DB).

\begin{tabular}{|c|c|c|c|c|c|c|}
\hline \multirow{2}{*}{ Espécie } & \multirow{2}{*}{ Lenho } & \multicolumn{3}{|c|}{ Retratibilidade } & \multirow{2}{*}{$\mathrm{CA}$} & \multirow[b]{2}{*}{ DB $\left(\mathrm{g} / \mathrm{cm}^{3}\right)$} \\
\hline & & $\operatorname{Tg}(\%)$ & $\operatorname{Rd}(\%)$ & Vol $(\%)$ & & \\
\hline \multirow{2}{*}{ Eucalyptus camaldulensis } & Oposto & 8,9 & 5,6 & 15,7 & 1,6 & 0,671 \\
\hline & Tração & 9,3 & 7,0 & 16,4 & 1,5 & 0,657 \\
\hline \multirow{2}{*}{ Eucalyptus maculata } & Oposto & 10,1 & 6,7 & 17,8 & 1,5 & 0,743 \\
\hline & Tração & 10,5 & 7,6 & 18,5 & 1,5 & 0,755 \\
\hline \multirow{2}{*}{ Eucalyptus pilularis } & Oposto & 10,1 & 7,6 & 19,0 & 1,3 & 0,714 \\
\hline & Tração & 10,3 & 6,5 & 17,2 & 1,7 & 0,686 \\
\hline \multirow{2}{*}{ Eucalyptus urophylla } & Oposto & 10,9 & 4,7 & 16,6 & 2,3 & 0,576 \\
\hline & Tração & 10,0 & 4,5 & 15,8 & 2,3 & 0,567 \\
\hline
\end{tabular}

Observa-se para a contração tangencial, que três espécies apresentaram-se maiores no lenho de tração e apenas o E. urophylla apresentou contração 8,25\% superior no lenho oposto. Para a contração radial e contração volumétrica duas espécies (E. camaldulensis e E. maculata) foram superiores no lenho de tração e as outras duas espécies foram superiores no lenho oposto. Para o E. camaldulensis a contração radial foi $20 \%$ superior no lenho de tração. No coeficiente de anisotropia duas espécies foram idênticas ( $E$. maculata e E. urophylla) e as duas outras espécies não apresentaram comportamento semelhante entre os lenhos. Sendo que para E. pilularis o coeficiente de anisotropia foi $23,5 \%$ superior no lenho de tração. Analisando a densidade básica apenas a espécie E. maculata apresentou aumento no lenho de tração tendo as outras três espécies apresentado aumento no lenho oposto.

\section{RESUMO}

A madeira de reação é formada na tentativa da árvore permanecer na posição vertical como reação à ação de forças como ventos, copa irregular ou inclinação do terreno que tendem a incliná-la. Em folhosas, como nos Eucalyptus, este tipo de madeira recebe o nome de madeira de tração e ocorre no lado da ação da força. 
Um indicativo da presença deste tipo de madeira é a elevada retratibilidade e densidade básica quando comparada a madeira normal. Sendo a densidade básica juntamente com a retratibilidade parâmetros para determinação da qualidade da madeira, o presente trabalho teve como objetivo avaliar a variação da densidade básica e da retratibilidade dos lenhos de tração e oposto ao longo do raio em quatro espécies de Eucalyptus spp. Quatro árvores das espécies Eucalyptus camaldulensis, Eucalyptus maculata, Eucalyptus pilularis e Eucalyptus urophylla, com 32 anos de idade, foram retiradas de um plantio experimental da Universidade Federal de Lavras. Amostras foram confeccionadas amostrando a extensão diametral nos lenhos de tração e oposto de discos do DAP. Os resultados indicam que as propriedades contração radial, contração tangencial, contração volumétrica, coeficiente de anisotropia e densidade básica não diferiram estatisticamente entre o lenho de tração e o lenho oposto.

Palavras-chave: Densidade básica, retratibilidade, lenho de reação, variação medula casca

\section{CONCLUSÃO}

As propriedades contração radial, contração tangencial, contração volumétrica, coeficiente de anisotropia e densidade básica não diferiram estatisticamente entre o lenho de tração e o lenho oposto nos Eucalyptus com 32 anos.

\section{REFERÊNCIAS}

ABNT- Associação Brasileira de Normas Técnicas. NBR 11941. Brasília, 2003. 6p.

Cruz, C. R.; Lima, J. T.; Muniz, G. I. B. (2003), Variações dentro das árvores e entre clones das propriedades físicas e mecânicas da madeira de híbridos de Eucalyptus. Scientia Forestalis, 64, 33-47.

Durlo, M. A.; Marchiori, J. N. C. (1992), Tecnologia da madeira: retratibilidade. Santa Maria: CEPEF/FATEC, (Série técnica, 10), 33p.

Ferreira, S. Lenho de tração em Eucalyptus spp cultivados em diferentes topografias. Tese (Doutorado em Ciências e Tecnologia da Madeira) - Universidade Federal de Lavras, 2007.

Ferreira, S.; Lima, J. T.; Trugilho, P. F.; Monteiro, T. C. (2008), Excentricidade da medula em caules de clones de Eucalyptus cultivados em diferentes topografias. Revista Cerne, 14, 335-340.

Green, D. W.; Winandy, J. E.; Kretschmann, D. E. (1999), Mechanical Properties of Wood. In: Wood handbook - Wood as an engineering material. Gen. Tech. Rep. FPL-GTR-113. Madison, W. I.: U.S. Department of Agriculture, Forest Service, Forest Products Laboratory, Cap 4, 1-45.

Panshin, A. J. and De Zeeuw, C. (1980), Text book of wood technology. 4.ed. New York: McGraw-Hill, 722p.

Ponce, R. H. e Watai, L. T. (1985), Manual de secagem da Madeira. MIC/STI/IPT. Brasilia, 70p.

Ruelle, J. M.; Yoshida, B.; Clair \& B. Thibaut. (2007), Peculiar tension wood structure in Laetia procera (Poepp.) Eichl. (Flacourtiaceae). Trees, 21, 345-355.

Simpson, W. and Tenwolde, A. (1999), Physical Properties and Moisture Relations of Wood. In: Wood handbook - Wood as an engineering material. Gen. Tech. Rep. FPL-GTR-113. Madison, WI: U.S. Department of Agriculture, Forest Service, Forest Products Laboratory, Cap 3, 1-24.

Sousa, L. C. Caracterização da madeira de tração em Eucalyptus grandis e sua influência na produção de polpa celulósica. Dissertação (Mestrado Ciência Florestal) - Universidade Federal de Viçosa, 2004.

Tomazello Filho, M. (1985), Variação radial da densidade básica e da estrutura anatômica da madeira do Eucalyptus saligna e E. grandis. IPEF, 29, 37-45.

Yi-Quing, W. ; Kazuo, H. ; Yuan, L. ; Yingchun, C. ; Masatoshi, S. (2006), Relationships of anatomical characteristics versus shrinkage and collapse properties in plantation-grown eucalypt wood from China. Journal of Wood Science, 52, 187-194.

Zobel, B. J. and Jett, J. B. (1995), Genetics of wood production. New York: Springer -Verlag, $337 \mathrm{p}$. 